DOI: https://doi.org/10.47405/mjssh.v6i1.629

\begin{tabular}{|c|c|}
\hline 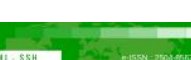 & Malaysian Journal of Social Sciences and Humanities (MJSSH) \\
\hline Malaysian Journal of & Volume 6, Issue 1, January 2021 \\
\hline (Mul-SSH) & e-ISSN : 2504-8562 \\
\hline & $\begin{array}{l}\text { Journal home page: } \\
\text { www.msocialsciences.com }\end{array}$ \\
\hline
\end{tabular}

\title{
Gender Differences in Attitude Towards Pornography Among Youth in Kelantan
}

\author{
Norazlifah Muhamad', Azriani Ab Rahman', Mohd Ismail Ibrahim¹, Razlina Abdul Rahman², \\ Wan Nor Arifin Wan Mansor ${ }^{3}$, Mokhtarrudin Ahmad4 \\ ${ }^{1}$ Department of Community Medicine, School of Medical Sciences, Universiti Sains Malaysia (USM) \\ 2Department of Family Medicine, School of Medical Sciences, Universiti Sains Malaysia (USM) \\ ${ }^{3}$ Unit of Biostatistics and Research Methodology, School of Medical Sciences, Universiti Sains Malaysia (USM) \\ ${ }^{4}$ Faculty of Applied Communication, Multimedia University \\ Correspondence: Azriani Ab Rahman (azriani@usm.my)
}

\begin{abstract}
Although the number of pornography exposure is increasing, our understandings on the issues are still lacking. This study aimed to compare gender differences in the attitudes towards pornography among youth in Kelantan. A cross sectional study was conducted using a validated self-administered questionnaire (PORQUE) on 930 college students in both government and private colleges in Kelantan. The mean scores were significantly higher among females for majority of the items assessing nonpermissive feelings toward pornography materials $(p<0.001)$, perception on impact of pornography $(p<0.05)$ and perception on factors contributed to pornography $(p<0.05)$. The mean score for majority of the items assessing permissive and perceptions toward pornography materials were significantly higher among males $(\mathrm{p}<0.001)$. Females were more non-permissive towards pornography, better perceived the impact of pornography and factors contributed to pornography compared to males. These findings are important as it serve as a guide for the stakeholders to implement pornography intervention for youth in Malaysia.
\end{abstract}

Keywords: pornography, attitude, youth, gender

\section{Introduction}

Internet usage has been increasing since its introduction in 1996. According to Malaysian Communication and Multimedia Commission (MCMC), the levels of internet penetration and mobile phone usage by adolescents were 53.6\% (Commission, 2017). Study by (Marret \& Wan-Yuen, 2014) found that majority of adolescents had access to a mobile smart phone by the age of twelve. Thus, expansion of internet access via computer or smartphones was linked to an increase in pornography consumption (Wilburn, 2016). Furthermore, adolescents with greater sexual experience tend to use internet pornography more frequent (Peter \& Valkenburg, 2016). A Malaysian survey reported 12\% of children surfed porn website when parents are not around (Zulhuda, 2015). This is thought to be due to easily accessibility of internet in cybercafé, free Wi-Fi in restaurants or affordable internet package or inexpensive devise (Commission, 2017).

Most studies had demonstrated negative outcomes of pornography such as premarital sexual activity (Johansson \& Hammaren, 2007), sex addiction (Peter \& Valkenburg, 2008), sexual violence such as rape (Morgan, 2011), viewing women as sex objects (Peter \& Valkenburg, 2007), sexual abuse and 
pedophilia (Marret \& Choo, 2018), relationship problems such as reduce sexual intimacy and infidelity (Flood, 2009a).

Pornography is defined as materials that are sexually explicit (SEM), which intended to create sexual arousal in the consumers (Habidin, Abdullah \& Salleh). SEM includes genitals or sexual activities such as masturbation, oral sex, vaginal and anal penetration in unconcealed way (Peter \& Valkenburg, 2016). Pornography is still considered as a taboo especially in a majority Muslim country that still uphold modesty and positive values such as Malaysia. Nevertheless, it has penetrated into the society as the materials are easily accessible through mobile phones or internet since the past two decades (Gnasigamoney \& Sidhu, 2013).

In Malaysia, any form of obscene materials are considered illegal and thus enforced by different acts such as The Printing Presses and Publications Act 1998 (PPPA), the Film Censorship Act 2002 (FCA), Penal Code, Communication and Multimedia Act 1998 (CMA) and Content Code (CC) (Zulhuda, 2015).

Pornography attitudes have been studied in majority countries and produced consistent result. Most studies reported negative or non-permissive attitudes toward pornography (Flood, 2009b; Wolak, Mitchell, \& Finkelhor, 2007). Most of the negative attitudes towards pornography occurred with unintentional exposure and involved younger ages. However, some adolescents were upset with the exposure on pornography for fear of their parents and not due to the contents (Flood, 2009a). When gender is compared, females especially the youngest age group reported more negative attitudes e.g. "degrading", "disgusting", felt "sick", "shocked", "embarrassed", "repulse" and "upset" compared to males (especially in the younger age group) who described pornography as "stimulating", "cool" or "exciting" (Wallmyr \& Welin, 2006). A study by (Marret \& Wan-Yuen, 2014) in Klang Valley revealed that girls who came across pornography websites by accident were distressed by what they saw.

Despite the increasing negative evidence and the consequences of pornography, study which specifically focused on attitude towards pornography is still lacking. The aim of this study is to compare gender differences in the attitudes towards pornography among youth in Kelantan.

\section{Methodology}

\section{Study design, study participants and sampling method}

This cross-sectional study was conducted among youth studying in colleges in Kelantan. This study was conducted upon approval by the ethics committee of Universiti Sains Malaysia (JEPeM Code: USM/JEPeM/17110604). Permission from the principles or directors of the selected colleges were obtained.

Simple random sampling was used to select ten out of 14 colleges from ten districts in Kelantan. The selection of the ten colleges was done randomly by using a software (Research Randomizer). Initially, the numbers of eligible classes were gathered from each college. Eligible classes referred to all the classes with students who fulfilled the inclusion and exclusion criteria (eligible students). The inclusion criteria were youth aged 16 to 24 whereas exclusion criteria were those who did not understand Malay language, non-Malaysian and those who didn't attend classes within the period of data collection. The classes were then randomly selected using a software (Research Randomizer) based on number of students required, which was proportionate to the total number of students from each institution.

\section{Research Tools}

A validated questionnaire on pornography (PORQUE)(Ab Rahman, Ibrahim, Rahman, Arifin, \& Ahmad, 2020) was used. The self-administered questionnaires were divided into two sections. The first 
section consisted of questionnaire on sociodemographic factors, family background, housing condition, religious practice, personal risk behavior and internet use. The second section comprised of attitude questionnaires of 5 domains: Non permissive feeling towards pornography (14 items) (Cronbach's alpha $=0.966$ ), permissive feeling towards pornography (6 items) (Cronbach's alpha $=0.929)$, permissive perception towards pornography (14 items) $(\alpha=0.924)$, perception on impact of pornography use (11 items) (Cronbach's alpha $=0.907)$ and perception on factors contributed to pornography exposure $(11$ items) (Cronbach's alpha=0.904). It encompassed a 5-point Likert scale scoring system ranging from $1=$ strongly disagree, $2=$ disagree, $3=$ not sure, $4=$ agree and $5=$ strongly agree. Higher score indicates non-permissive attitude towards pornography for 'non permissive feeling towards pornography materials', 'perception on impact of pornography use' and 'perception on factors contributed to pornography exposure' domains whereas lower score indicates permissive attitude towards pornography for 'permissive feeling towards pornography materials' and 'permissive perception towards pornography materials' domains.

\section{Data Collection}

All eligible students in the selected classes were invited to participate in this study and were given consent forms prior to data collection. Data collection was done only after they agreed to participate in this study. Students were provided with explanations for some terminologies stated in the questionnaires such as 'pornography', 'sex education' and 'pedophilia'. The questionnaires were selfadministered and anonymous to ensure confidentiality. All the completed questionnaires were collected and kept in a sealed envelope by the researchers after the completion of each session.

\section{StatisticalAnalysis}

The data were analyzed using IBM SPSS statistics version 24. Independent T-test was used for analysis of gender difference in attitudes.

\section{Result}

\section{Sociodemographic and internet use}

Out of 930 respondents, 71 were excluded due to major incomplete response i.e., didn't complete any section of the questionnaire. We allowed a few missing data to be included in our analysis as we didn't record any name or contact number of our respondents for clarification purpose (to maintain the confidentiality). Overall, the response rate was $92.4 \%$.

The mean age of respondents was 19.7(SD1.3) years with female to male ratio was almost 1:1. Majority of them were Malays (98.5\%). In terms of internet use, almost all of our students claimed that they received internet access via mobile phones and used internet on average 6 hours a day. The most commonly internet application used by our respondents was Instagram (77.9\%), followed by Facebook (69.6\%), Youtube (59.1\%), Wechat (22.5\%), Twitter (17.2\%) and Telegram (10.4\%). Majority of them claimed that they regularly surfed the internet alone (83.5\%) (Table 1$)$.

Table 1: Sociodemographic factors and internet use of the respondents

\begin{tabular}{lcc}
\hline Variables & Mean (SD) & n (\%) \\
\hline Socio-demographic & & \\
Age (year) & $19.7(1.3)$ & \\
Gender (missing data n=1) & \\
$\quad$ Male & & $389(45.3)$ \\
$\quad$ Female & $469(54.7)$ \\
Race & & \\
$\quad$ Malay & $846(98.5)$
\end{tabular}


Others

\section{Internet use}

Do you always use internet? (Missing data $n=3$ )

Yes

$840(98.1)$

No

$16(1.9)$

Medium of internet (missing data $n=5$ )

Mobile phone

Yes

No

Computer

Yes

No

Duration of internet use per day (hours)

$5.9(6.2)$

Commonly used application

Facebook (missing data $\mathrm{n}=3$ )

Yes

$596(69.6)$

No

$260(30.4)$

Instagram (missing data $\mathrm{n}=3$ )

Yes

667 (77.9)

No

$189(22.1)$

WeChat (missing data $n=3$ )

Yes

$193(22.5)$

No

$663(77.5)$

Telegram ( missing data $n=4)$

Yes

$89(10.4)$

No

$766(89.6)$

Twitter (missing data $\mathrm{n}=4$ )

Yes

$147(17.2)$

No

$708(82.8)$

You Tube (missing data $n=6$ )

$504(59.1)$

Yes

349 (40.9)

Internet surfing accompany (missing data $\mathrm{n}=7$ )

Alone

Yes

$711(83.5)$

No

$141(16.5)$

\section{Attitude scores of each domain}

The lowest mean score was noted for permissive feeling towards pornography materials and the highest mean score was noted for non-permissive feeling towards pornography materials as shown in Table 2 .

Table 2: Mean Score for Attitude on Pornography

\begin{tabular}{lc}
\hline Domains for attitude questionnaire & Mean (SD) \\
\hline 1. Non permissive feeling towards pornography materials & $52.9(10.8)$ \\
2. Permissive feeling towards pornography materials & $13.3(6.4)$ \\
3. Permissive perception towards pornography materials & $28.1(11.5)$ \\
4. Perception on impact of pornography use & $46.3(8.6)$ \\
5. Perception on factors contributed to pornography exposure & $47.3(7.1)$ \\
\hline
\end{tabular}


DOI: https://doi.org/10.47405/mjssh.v6i1.629

\section{Gender Difference in Attitude towards Pornography}

For non-permissive feelings toward pornography materials domain, the mean score were significantly higher among females for all items $(p<0.001)$ except for Q1 and Q2 (Table 3).

Table 3: Mean Score Between Gender for Non-Permissive Feelings Toward Pornography Materials

\begin{tabular}{llllll}
\hline Items & $\begin{array}{l}\text { Male } \\
(\mathbf{n = 3 8 9})\end{array}$ & $\begin{array}{l}\text { Female } \\
(\mathbf{n = 4 6 9 )}\end{array}$ & $\mathbf{t}$ & df & p-value \\
\cline { 2 - 5 } & Mean (SD) & Mean (SD) & & & \\
\hline $\begin{array}{l}\text { Q1: I feel disturbed after reading } \\
\text { pornography materials }\end{array}$ & $3.44(1.17)$ & $3.38(1.24)$ & 0.79 & 848 & 0.688 \\
\hline $\begin{array}{l}\text { Q2: I feel disturbed after watching } \\
\text { pornography materials }\end{array}$ & $3.46(1.20)$ & $3.39(1.26)$ & 0.84 & 848 & 0.731 \\
\hline $\begin{array}{l}\text { Q3: I feel shy after reading } \\
\text { pornography materials }\end{array}$ & $3.56(1.09)$ & $4.05(1.05)$ & -6.55 & 805.5 & $<0.001$ \\
\hline $\begin{array}{l}\text { Q4: I feel shy after watching } \\
\text { pornography materials }\end{array}$ & $3.59(1.07)$ & $4.05(1.05)$ & -6.23 & 812.6 & $<0.001$ \\
\hline $\begin{array}{l}\text { Q5: I feel shock when accidentally } \\
\text { read pornography materials }\end{array}$ & $3.35(1.11)$ & $4.11(1.0)$ & - & 780.9 & $<0.001$ \\
\hline $\begin{array}{l}\text { Q6: I feel palpitation after } \\
\text { watching pornography materials }\end{array}$ & $3.39(1.12)$ & $3.72(1.19)$ & -4.17 & 843 & $<0.001$ \\
\hline $\begin{array}{l}\text { Q7 I feel depress after reading } \\
\text { pornography materials }\end{array}$ & $3.08(1.08)$ & $3.42(1.18)$ & -4.39 & 837.9 & $<0.001$ \\
\hline $\begin{array}{l}\text { Q8: I feel depress after watching } \\
\text { pornography materials }\end{array}$ & $3.08(1.08)$ & $3.41(1.21)$ & -4.31 & 841.3 & $<0.001$ \\
\hline $\begin{array}{l}\text { Q9: I feel guilty after reading } \\
\text { pornography materials }\end{array}$ & $3.76(1.03)$ & $4.20(1.03)$ & -6.24 & 846 & $<0.001$ \\
\hline $\begin{array}{l}\text { Q10: I feel guilty after watching } \\
\text { pornography materials }\end{array}$ & $3.81(1.06)$ & $4.25(1.01)$ & -6.21 & 846 & $<0.001$ \\
\hline $\begin{array}{l}\text { Q11: I feel gross after accidentally } \\
\text { read pornographic materials }\end{array}$ & $3.55(1.08)$ & $4.27(1.0)$ & -9.97 & 791.2 & $<0.001$ \\
\hline $\begin{array}{l}\text { Q12: I feel gross after accidentally } \\
\text { read pornographic materials }\end{array}$ & $3.59(1.10)$ & $4.30(0.99)$ & -9.80 & 782.9 & $<0.001$ \\
\hline $\begin{array}{l}\text { Q13: I feel sinful after reading } \\
\text { pornographic materials }\end{array}$ & $4.19(0.98)$ & 4.44 & -3.89 & 846 & $<0.001$ \\
\hline $\begin{array}{l}\text { Q14: I feel sinful after watching } \\
\text { pornographic materials }\end{array}$ & $4.19(1.01)$ & $4.44(0.98)$ & -3.60 & 846 & $<0.001$ \\
\hline a Independent T-test & & & & & \\
\hline
\end{tabular}

The mean score for all items of permissive feelings toward pornography materials domain were significantly higher among male $(p<0.001)$ (table 4$)$.

Table 4: Mean Score between Gender for Permissive Feelings toward Pornography Materials

\begin{tabular}{llllll}
\hline \multirow{2}{*}{ Item } & $\begin{array}{l}\text { Male } \\
(\mathbf{n = 3 8 9})\end{array}$ & $\begin{array}{l}\text { Female } \\
(\mathbf{n = 4 6 9 )}\end{array}$ & t & df & p-value \\
\cline { 2 - 4 } & Mean (SD) & Mean (SD) & & & \\
\hline $\begin{array}{l}\text { Q15: I feel excited after } \\
\text { reading pornographic } \\
\text { materials }\end{array}$ & $2.47(1.17)$ & $1.84(1.14)$ & 7.94 & 845 & $<0.001$ \\
\hline
\end{tabular}


DOI: https://doi.org/10.47405/mjssh.v6i1.629

\begin{tabular}{llllll}
\hline $\begin{array}{l}\text { Q16: I feel excited after } \\
\text { watching pornographic } \\
\text { materials }\end{array}$ & $2.42(1.15)$ & $1.83(1.15)$ & 7.42 & 845 & $<0.001$ \\
\hline $\begin{array}{l}\text { Q17: I feel passionate } \\
\text { after reading } \\
\text { pornographic materials }\end{array}$ & $2.96(1.19)$ & $2.07(1.21)$ & 10.73 & 845 & $<0.001$ \\
\hline $\begin{array}{l}\text { Q18: I feel passionate } \\
\text { after watching } \\
\text { pornographic materials }\end{array}$ & $2.96(1.19)$ & $2.07(1.22)$ & 10.73 & 845 & $<0.001$ \\
\hline $\begin{array}{l}\text { Q19: I feel like trying } \\
\text { sexual acts after reading } \\
\text { pornographic materials }\end{array}$ & $2.96(1.19)$ & $1.70(1.08)$ & 9.49 & 782 & $<0.001$ \\
\hline $\begin{array}{l}\text { Q20: I feel like trying } \\
\text { sexual acts after } \\
\text { watching pornographic } \\
\text { materials }\end{array}$ & $2.49(1.22)$ & $1.69(1.08)$ & 10.03 & 773.9 & $<0.001$ \\
\hline a Independent T-test & & & & \\
\hline
\end{tabular}

Table 5 shows the mean score for all items of permissive perceptions toward pornography materials domain were significantly higher among male $(p<0.001)$

Table 5: Mean Score Between Gender for Permissive Perceptions Toward Pornography Materials

\begin{tabular}{|c|c|c|c|c|c|}
\hline \multirow[t]{2}{*}{ Item } & Male $(n=389)$ & $\begin{array}{l}\begin{array}{l}\text { Female } \\
(n=469)\end{array} \\
\end{array}$ & $\bar{t}$ & \multirow{2}{*}{\multicolumn{2}{|c|}{ p-value ${ }^{a}$}} \\
\hline & Mean (SD) & Mean (SD) & & & \\
\hline $\begin{array}{l}\text { Q21: Reading pornographic } \\
\text { materials is not wrong }\end{array}$ & $2.17(1.17)$ & $\begin{array}{l}1.81 \\
(1.03) \\
\end{array}$ & 4.66 & 767.7 & $<0.001$ \\
\hline $\begin{array}{l}\text { Q22: Watching pornography is } \\
\text { not wrong }\end{array}$ & $2.06(1.16)$ & $\begin{array}{l}1.69 \\
(0.95)\end{array}$ & 4.98 & 733.8 & $3<0.001$ \\
\hline $\begin{array}{l}\text { Q23: Reading pornographic } \\
\text { materials is important for a } \\
\text { teenager like me }\end{array}$ & $2.20(1.04)$ & $\begin{array}{l}1.66 \\
(0.88)\end{array}$ & 8.01 & 749.6 & $5<0.001$ \\
\hline $\begin{array}{l}\text { Q24: Watching pornographic } \\
\text { materials is important for a } \\
\text { teenager like me }\end{array}$ & $2.13(1.05)$ & $\begin{array}{l}1.62 \\
(0.86)\end{array}$ & 7.55 & 734.7 & $<0.001$ \\
\hline $\begin{array}{l}\text { Q25: I can easily understand the } \\
\text { contents from pornographic } \\
\text { materials than sex education at } \\
\text { schools }\end{array}$ & $2.43(1.06)$ & $\begin{array}{l}1.77 \\
(0.95)\end{array}$ & 9.47 & 776 & $<0.001$ \\
\hline $\begin{array}{l}\text { Q26: I wish to know more } \\
\text { information on sex by reading } \\
\text { pornographic materials }\end{array}$ & $2.33(1.07)$ & $\begin{array}{l}1.66 \\
(0.84)\end{array}$ & 9.89 & 713.7 & $7<0.001$ \\
\hline $\begin{array}{l}\text { Q27: I wish to know more } \\
\text { information on sex by watching } \\
\text { pornographic materials }\end{array}$ & $2.31(1.06)$ & $\begin{array}{l}1.62 \\
(0.81)\end{array}$ & 10.28 & 705.4 & $<0.001$ \\
\hline $\begin{array}{l}\text { Q28: My knowledge on sex } \\
\text { increase after reading } \\
\text { pornographic materials }\end{array}$ & $2.52(1.12)$ & $\begin{array}{l}1.86 \\
(1.01)\end{array}$ & 8.75 & 792.1 & $<0.001$ \\
\hline $\begin{array}{l}\text { Q29: My knowledge on sex } \\
\text { increase after watching } \\
\text { pornographic materials }\end{array}$ & $2.59(1.12)$ & $\begin{array}{l}1.83 \\
(1.01)\end{array}$ & 10.24 & 776.9 & $<0.001$ \\
\hline $\begin{array}{l}\text { Q30: I can generate income } \\
\text { from activities related to } \\
\text { pornography }\end{array}$ & $1.94(0.99)$ & $\begin{array}{l}1.44 \\
(0.73)\end{array}$ & 8.12 & 686.4 & $t<0.001$ \\
\hline
\end{tabular}



DOI: https://doi.org/10.47405/mjssh.v6i1.629

\begin{tabular}{llllll}
\hline $\begin{array}{l}\text { Q31: I feel that reading } \\
\text { pornographic materials is better } \\
\text { than committing sexual act }\end{array}$ & $2.64(1.19)$ & $\begin{array}{l}1.96 \\
(1.08)\end{array}$ & 8.59 & 779.5 & $<0.001$ \\
\hline $\begin{array}{l}\text { Q32: I feel that watching } \\
\text { pornographic materials is better } \\
\text { than committing sexual act }\end{array}$ & $2.67(1.18)$ & $\begin{array}{l}1.91 \\
(1.07)\end{array}$ & 9.84 & 842 & $<0.001$ \\
\hline $\begin{array}{l}\text { Q33: Reading pornographic } \\
\text { materials can release stress }\end{array}$ & $2.49(1.13)$ & $\begin{array}{l}1.59 \\
(0.83)\end{array}$ & 12.86 & 684.4 & $<0.001$ \\
\hline $\begin{array}{l}\text { Q34: Watching pornographic } \\
\text { materials can release stress }\end{array}$ & $2.49(1.16)$ & $\begin{array}{l}1.58 \\
(0.82)\end{array}$ & 12.93 & 670.8 & $<0.001$ \\
\hline
\end{tabular}

${ }^{\mathrm{a}}$ Independent T-test

The mean score for all the items of perception on impact of pornography use domain (excluding Q35 and Q36) were significantly higher among female $(p<0.05)$ (Table 6$)$.

Table 6: Mean Score Between Gender for Perception on Impact of Pornography Use

\begin{tabular}{|c|c|c|c|c|c|}
\hline Items & $\begin{array}{l}\text { Male } \\
(\mathrm{n}=389)\end{array}$ & $\begin{array}{l}\text { Female } \\
(n=469)\end{array}$ & $\mathbf{t}$ & df & p-value ${ }^{a}$ \\
\hline & Mean (SD) & Mean (SD) & & & \\
\hline $\begin{array}{l}\text { Q35: Pornography is the } \\
\text { cause of illegitimate child }\end{array}$ & $4.09(1.02)$ & $4.23(0.95)$ & -2.10 & 840 & 0.251 \\
\hline $\begin{array}{l}\text { Q36: Pornography is the } \\
\text { cause of pre-marital } \\
\text { pregnancy }\end{array}$ & $4.10(1.00)$ & $4.26(0.92)$ & -2.40 & 840 & 0.180 \\
\hline $\begin{array}{l}\text { Q37: Pornography is the } \\
\text { cause of free sex }\end{array}$ & $4.16(0.93)$ & $4.36(0.81)$ & -3.33 & 840 & 0.006 \\
\hline $\begin{array}{l}\text { Q38 Pornography is the } \\
\text { cause of paedophilia }\end{array}$ & $4.07(0.97)$ & $4.29(0.84)$ & -3.52 & 840 & 0.012 \\
\hline $\begin{array}{l}\text { Q39: Pornography is the } \\
\text { cause of LGBT }\end{array}$ & $3.98(0.95)$ & $4.24(0.84)$ & -4.16 & 840 & 0.001 \\
\hline $\begin{array}{l}\text { Q40: Pornography is the } \\
\text { cause of premarital sex }\end{array}$ & $4.14(0.92)$ & $4.35(0.79)$ & -3.55 & 840 & 0.007 \\
\hline $\begin{array}{l}\text { Q41: Pornography is the } \\
\text { cause of rape cases }\end{array}$ & $4.14(0.92)$ & $4.40(0.78)$ & -4.31 & 840 & $<0.001$ \\
\hline $\begin{array}{l}\text { Q42: Pornography is the } \\
\text { cause of sex addiction }\end{array}$ & $4.16(0.91)$ & $4.37(0.78)$ & -3.63 & 840 & 0.003 \\
\hline $\begin{array}{l}\text { Q43: Pornography is the } \\
\text { cause of sex violence }\end{array}$ & $4.10(0.93)$ & $4.32(0.78)$ & -3.72 & 840 & 0.004 \\
\hline $\begin{array}{l}\text { Q44: Exposure to } \\
\text { pornography is the cause of } \\
\text { women, men and children } \\
\text { being regarded as sex objects }\end{array}$ & $4.03(0.96)$ & $4.25(0.83)$ & -3.64 & 840 & 0.007 \\
\hline $\begin{array}{l}\text { Q45: Pornography is the } \\
\text { cause of sex exploitation in } \\
\text { men/women/children }\end{array}$ & $3.99(0.95)$ & $4.24(0.83)$ & -4.11 & 840 & 0.002 \\
\hline
\end{tabular}

The mean score for seven out of 11 items of perception on factors contributed to the use of pornography exposure domain were significantly higher among female (Q46, Q49, Q51, Q52, Q54, Q55, Q56) with $p<0.05$. (Table 7). 
Table 7: Mean Score Between Gender for Perception on Factors Contributed to The Use of Pornography Exposure

\begin{tabular}{|c|c|c|c|c|c|}
\hline Items & $\begin{array}{l}\begin{array}{l}\text { Male } \\
(\mathrm{n}=389)\end{array} \\
\text { Mean } \\
\text { (SD) }\end{array}$ & $\begin{array}{l}\begin{array}{l}\text { Female } \\
(\mathrm{n}=469)\end{array} \\
\text { Mean } \\
(\text { SD) }\end{array}$ & $\mathbf{t}$ & df & p-value ${ }^{a}$ \\
\hline $\begin{array}{l}\text { Q46: Parents monitoring on } \\
\text { internet use can prevent exposure } \\
\text { to pornography }\end{array}$ & $\begin{array}{l}4.32 \\
(0.84)\end{array}$ & $4.50(0.72)$ & -3.31 & 753.9 & $\mathbf{0 . 0 0 3}$ \\
\hline $\begin{array}{l}\text { Q47: Parents monitoring on mobile } \\
\text { phone use can prevent exposure to } \\
\text { pornography }\end{array}$ & $\begin{array}{l}4.35 \\
(0.79)\end{array}$ & $4.49(0.72)$ & -2.69 & 776.2 & 0.056 \\
\hline $\begin{array}{l}\text { Q48: Lack of religious education is } \\
\text { the cause of pornography use }\end{array}$ & $\begin{array}{l}4.38 \\
(0.81)\end{array}$ & $4.47(0.72)$ & -1.56 & 771.3 & 0.111 \\
\hline $\begin{array}{l}\text { Q49: Lack of family ties is the } \\
\text { cause of pornography use }\end{array}$ & $\begin{array}{l}4.09 \\
(0.92)\end{array}$ & $4.27(0.82)$ & -2.97 & 842 & 0.042 \\
\hline $\begin{array}{l}\text { Q50: Lack of religious practice is } \\
\text { the cause of pornography use }\end{array}$ & $\begin{array}{l}4.38 \\
(0.83)\end{array}$ & $4.45(0.72)$ & -1.13 & 760.6 & 0.244 \\
\hline $\begin{array}{l}\text { Q51: Lack of religiosity/moral } \\
\text { values is the cause of pornography } \\
\text { use }\end{array}$ & $\begin{array}{l}4.38 \\
(0.83)\end{array}$ & $4.45(0.71)$ & -1.21 & 753.7 & 0.039 \\
\hline $\begin{array}{l}\text { Q52: Extensive internet use is the } \\
\text { cause of pornography widespread }\end{array}$ & $\begin{array}{l}4.27 \\
(0.86)\end{array}$ & $4.44(0.73)$ & -2.93 & 746.7 & 0.006 \\
\hline $\begin{array}{l}\text { Q53: Sex education at schools can } \\
\text { reduce the use of pornography } \\
\text { materials }\end{array}$ & $\begin{array}{l}3.64 \\
(1.10)\end{array}$ & $3.69(1.13)$ & -0.54 & 841 & 0.476 \\
\hline $\begin{array}{l}\text { Q54: Parents who spend more time } \\
\text { with their kids can reduce the use } \\
\text { of pornography materials among } \\
\text { their kids }\end{array}$ & $\begin{array}{l}4.30 \\
(0.84)\end{array}$ & $4.47(0.71)$ & -3.10 & 749.4 & 0.031 \\
\hline $\begin{array}{l}\text { Q55: Parents who are close with } \\
\text { their kids can reduce the use of } \\
\text { pornography materials among their } \\
\text { kids }\end{array}$ & $\begin{array}{l}4.27 \\
(0.84)\end{array}$ & $4.45(0.71)$ & -3.33 & 749.2 & 0.012 \\
\hline $\begin{array}{l}\text { Q56: Family disharmony is a cause } \\
\text { for use of pornography materials } \\
\text { among their kids }\end{array}$ & $\begin{array}{l}4.06 \\
(0.95)\end{array}$ & $4.22(0.84)$ & -2.60 & 842 & 0.017 \\
\hline
\end{tabular}

\section{Discussion}

Result for the attitudes questionnaire indicated higher score for non-permissive (or negative) attitude and vice versa for permissive (or positive) attitude toward pornography. Domain with the highest mean score was 'non permissive feeling towards pornography materials' whereas domain with the lowest mean score was 'permissive feeling towards pornography materials. This finding was consistent with previous studies (Emmers-Sommer, Hertlein, \& Kennedy, 2013; Flood, 2009a; Johansson \& Hammaren, 2007; Mitchell, Wolak, \& Finkelhor, 2007; Wallmyr \& Welin, 2006).

There were a few differences in the pornography attitudes when gender is compared. For example, female have more of non-permissive feelings toward pornography compared to male. They are more 'shy', 'shocked', 'had palpitations', 'depressed', and 'felt guilty', 'grossed' and 'sinful' after watching or reading pornography materials. On the other hand, male have more permissive feelings toward 
pornography materials compared to female. For example, males are shown to be more 'excited', more 'passionate' and had more tendency to try sexual act after reading or watching pornographic materials compared to female. These_differences in gender attitudes on pornography were consistent with a few findings from previous literature (Peter \& Valkenburg, 2008; Wallmyr \& Welin, 2006). However, other studies reported that both gender had permissive attitude towards pornography (Emmers-Sommer et al., 2013; Mattebo, Tydén, Häggström-Nordin, Nilsson, \& Larsson, 2014; Wijaya Mulya \& Hald, 2014).

Meanwhile, pornography often produced by men whom used women as the main player, thus attracted men more than women (Mattebo et al., 2014). Furthermore, men are more porno enthusiast, more experimental and more likely to focus on physical appearance for sexual attraction or sexual pleasure, which explained why men consumed pornography more than women (Emmers-Sommer et al., 2013). These statements were supported by our findings that males appeared to show more permissive perceptions toward pornographic materials. For example, males perceived that it was not wrong to read or watch pornography. They also perceived that watching or reading pornography was important and knowledge from pornography materials were easier to understand compared to sex education at schools. They also claimed that their knowledge increased after reading or watching pornographic materials, pornography can relieve their stress, and perceived that watching or reading pornography was better than committing sexual act. In reference to cultivation theory which suggest that frequent exposure to similar repetitive content will predict viewer's adoption of the subject, frequent exposure to pornography (deliberate or not) may result in 'adaptation' and subsequently 'normalization' of the behavior (Weinberg, Williams, Kleiner, \& Irizarry, 2010). Such 'normalization' may be further enhanced by peer pressure or wanting to be being accepted by the group. However, other factors such as age of first exposure, duration of exposure, religious background, parental guidance or pre-exposure sex education were a few factors that could determine the permissive perceptions as well which were not in our scope of study.

Female tend to perceive better in terms of impact of pornography compared to male. For example, some of the implications of pornography such as having illegitimate child, involving in free sex, pedophilia, LGBT, sex before marriage, rape cases, sex addiction and sex violence were better perceived by females than males. Females also seemed to have better mean score compared to males in terms of perception on factors contributed to pornography. For example, females perceived that good parental monitoring and good family relationship could reduce or prevent the intentional exposure to pornography whereas extensive internet use contribute to pornography use. As these findings seemed to fill in a study gap, it may also indicate females as a better recipient of sex education and better comprehend the negative effects of pornography or factors that contributed to pornography compared to men. This factor can be further explained by the fact that most of the impact of pornography such as pre-marital pregnancy, teenage pregnancy, domestic violence, women being regard as sex objects, having illegitimate child and raped cases falls on women.

We acknowledge a few limitations in our study. First, the result was limited to a certain age group (1624 years old). Thus, we were not able to determine the attitude of younger population towards pornography, which may be different to the older age group. Furthermore, our respondents were majority from Muslim, Malay group. Thus, this finding may not be generalized the other religion and race which may have different values and cultural assimilation. In addition, this study relies on selfreport measures, which unable for us to verify on the responses and may limit our interpretation due to missing data. Although the confidentiality of the study was ensured, there is still a probability of response bias as the respondents may know each other and thus shared the same ideas and thought about pornography.

\section{Conclusion}

Overall result proved that despite a widespread accessibility and easily availability of pornography in Malaysia, it is still regarded as negative or non-permissive attitudes by our respondents. However, men seemed to have more permissive attitudes and less perceived the impact on pornography and factors 
contributed to pornography. Thus, this study could assist the policy makers or stakeholders on different approach during intervention which tailored to specific gender.

\section{Acknowledgement}

The present study was funded by Tabung Insentif Pembangunan Pengajian Siswazah PPSP (TIPPS). The authors want to express our sincere gratitude to the principles and students involved in the study. We certify that there is no actual or potential conflict of interest in relation to this article.

\section{References}

Ab Rahman, A., Ibrahim, M. I., Rahman, R. A., Arifin, W. N., \& Ahmad, M. (2020). Development and Validation of a Malay Version of the Questionnaire on Pornography Attitudes and Exposure for Youth in Kelantan. The Malaysian Journal of Medical Sciences: MJMS, 27(2), 129.

Commission, M. C. a. M. (2017). Internet users survey 2017. Statistical Brief Number Twenty-One. ( 1823-2523).

Emmers-Sommer, T., Hertlein, K., \& Kennedy, A. (2013). Pornography use and attitudes: An examination of relational and sexual openness variables between and within gender. Marriage \& Family Review, 49(4), 349-365.

Flood, M. (2009a). The harms of pornography exposure among children and young people. Child Abuse Review: Journal of the British Association for the Study and Prevention of Child Abuse and Neglect, 18(6), 384-400.

Flood, M. (2009b). The Harms of Pornography Exposure Among Children and Young People. Child Abuse Review, 18, 384-400.

Gnasigamoney, S. S., \& Sidhu, M. S. (2013). E-behaviour trends and patterns among Malaysian preadolescents and adolescents. International Journal of Digital Crime and Forensics (IJDCF), $5(2), 50-62$.

Habidin, N., Abdullah, M. Y., \& Salleh, M. A. M. Online pornography on smartphones amongst the teenage juvenile. Humanities and Social Sciences Review,

CD-ROM. ISSN: 2165-6258::06(01):355-366 (2016).

Johansson, T., \& Hammaren, N. (2007). Hegemonic Masculinity and Pornography: Young people's attitudes toward and relations to pornography. The Journal of Men's Studies, 15(1), 57-70.

Marret, M. J., \& Choo, W.-Y. (2018). Victimization after meeting with online acquaintances: a crosssectional survey of adolescents in Malaysia. Journal of interpersonal violence, 33(15), 23522378 .

Marret, M. J., \& Wan-Yuen, C. (2014). Use of ICT by Minors: Key Project Findings. Kuala Lumpur: Universiti of Malaya.

Mattebo, M., Tydén, T., Häggström-Nordin, E., Nilsson, K. W., \& Larsson, M. (2014). Pornography and sexual experiences among high school students in Sweden. Journal of Developmental \& Behavioral Pediatrics, 35(3), 179-188.

Mitchell, K. J., Wolak, J., \& Finkelhor, D. (2007). Trends in youth reports of sexual solicitations, harassment and unwanted exposure to pornography on the Internet. Journal of adolescent health, 40(2), 116-126.

Morgan, E. M. (2011). Associations between young adults' use of sexually explicit materials and their sexual preferences, behaviors, and satisfaction. Journal of sex research, 48(6), 520-530.

Peter, J., \& Valkenburg, P. M. (2007). Adolescents' Exposure to a Sexualized Media Environment and Their Notions of Women as Sex Objects. Sex Roles, 56, 381-395.

Peter, J., \& Valkenburg, P. M. (2008). Adolescents' Exposure to Sexually Explicit Internet Material, Sexual Uncertainty, and Attitudes Toward Uncommitted Sexual Exploration:Is There a Link? Communication Research, 35(5), 579-601. doi:10.1177/0093650208321754

Peter, J., \& Valkenburg, P. M. (2016). Adolescents and pornography: a review of 20 years of research. The Journal of Sex Research, 53(4-5), 509-531.

Wallmyr, G., \& Welin, C. (2006). Young people, pornography, and sexuality: Sources and attitudes. The Journal of School Nursing, 22(5), 290-295. 
DOI: https://doi.org/10.47405/mjssh.v6i1.629

Weinberg, M. S., Williams, C. J., Kleiner, S., \& Irizarry, Y. (2010). Pornography, normalization, and empowerment. Archives of Sexual Behavior, 39(6), 1389-1401.

Wijaya Mulya, T., \& Hald, G. M. (2014). Self-perceived effects of pornography consumption in a sample of Indonesian university students. Media Psychology, 17(1), 78-101.

Wilburn, B. S. (2016). Examining the Effects of Pornography on the Individual and the Family. Eastern Illinois University.

Wolak, J., Mitchell, K., \& Finkelhor, D. (2007). Unwanted and wanted exposure to online pornography in a national sample of youth Internet users. Pediatrics, 119(2), 247-257.

Zulhuda, S. (2015). Cyberlaw on Pornography. Paper presented at the National Law Students Conferene Universiti Utara Malaysia. 